\title{
Circulating biomarkers of response to immunotherapy in cancer treatment
}

\author{
Marzia Del Re*,1(D), Giulia Gianfilippo', Stefania Crucitta', Alfredo Addeo², Ron HN van \\ Schaik ${ }^{3}$ \& Romano Danesi ${ }^{1}$ (iD \\ ${ }^{1}$ Unit of Clinical Pharmacology \& Pharmacogenetics, Department of Clinical \& Experimental Medicine, University Hospital of Pisa, \\ 56126, Italy \\ ${ }^{2}$ Oncology Department, University Hospital Geneva, Geneva 1205, Switzerland \\ ${ }^{3}$ Department of Clinical Chemistry, Erasmus University Medical Center, Rotterdam, South Holland 3015, The Netherlands \\ *Author for correspondence: marzia.delre@gmail.com
}
' ${ }_{\text {it }}$ is likely that a reliable predictive biomarker for immunotherapy will be a multivariate model, composed of TMB, TILs, PD-L1 expression not only in tissue, but also as circulating biomarkers to select and monitor patients during treatment"

First draft submitted: 6 August 2019; Accepted for publication: 11 September 2019; Published online: 6 November 2019

Keywords: circulating biomarkers $\bullet$ immunotherapy $\bullet$ predictive biomarkers $\bullet$ solid tumors

The introduction of checkpoint inhibitors in cancer has established durable clinical responses in patients affected by a variety of solid tumors. However, since only a subset of tumors and a small number of patients are responsive to these inhibitors, there are clinical concerns to be addressed in order to provide the optimal therapy. These factors involve aspects such as intratumor heterogeneity, individual immune system reactivity and innate or acquired drug resistance [1]. The patients' selection more likely to benefit from anti-PD-1 drugs is based on PD-L1 expression in tumor cells, especially in non-small-cell lung cancer (NSCLC) [2]. However, PD-L1 expression does not seem to be an optimal predictive biomarker, since some PD-L1-positive patients do not respond to therapy and some PD-L1-negative patients do experience benefit to some extent [3]. Therefore, the scientific community keeps looking for additional biomarkers, such as the tumor immune infiltrate scores (TILs), the mutational burden (TMB) and microsatellite instability (MSI) [4-6].

Recently, it has been demonstrated that high TMB correlates with high response rates to checkpoint inhibitors, probably due to the immunogenic neoantigens released and recognized as foreign by the immune system [6,7]. Moreover, MSI may also play a role in the immune response, since MSI tumors show high TMB with high response to immunotherapy across a wide range of tumor histology [8]. Among patients with the same TMB values, responses to checkpoints inhibitors may still differ, suggesting that the actual response to immunotherapy is more complex, and that it might depend on the quality of the neoantigen rather than the quantity and that additional mechanisms are involved [9]. All of the above-mentioned biomarkers are dependent on the availability of tissue samples, which represents a main constraint. Pharmacologic treatments may have an impact on tumors, dynamically modulating tumor heterogeneity through the treatment pressure. Sometimes this effect can be used in our favor by using therapies that are able to induce an immunogenic effect by the releasing of neoantigens derived from the death cells, inducing T-cell responses ('abscopal effect' - i.e., radiotherapy or specific chemotherapy agents) [10]. Sometimes treatment selective pressure just changes the molecular landscape of a tumor and immune response to tumors may undergo dynamic changes during the course of the disease [11,12].

Recent data have showed that mutation burden decreases accordingly to response in patients with melanoma receiving nivolumab, leading to a collapse of whole clonal populations in patients who respond, while selecting specific subclones in patients achieving a stable disease. In patients who received a prior line of immunotherapy, only the ones carrying a high PD-L1 expression or having an immunologically 'hot tumor' were more likely to respond. In patients with low or no PD-L1 expression, a significant response was achieved in the subgroup of patients treated with the combination of ipilimumab-nivolumab, suggesting that tumor response based on a 'hot 
tumor' may depend also on previous treatment [12]. Therefore, the analysis of tumor tissue has clear limitations and alternative approaches should be implemented to monitor the robustness of lymphocytic activation and the development of resistance mechanisms depending on tumor immune editing. Thus, studies on circulating biomarkers are increasing, providing important information on the dynamic response to treatments and relevant information on clinical outcome. The minimally invasive nature of the analysis of circulating biomarkers compared with the tumor tissue biopsy offers clear advantages, considering also that tissue availability may sometimes limit tissue testing. Circulating biomarkers can reflect the genomic heterogeneous landscape of a tumor, identifying prognostic and predictive biomarkers.

Clinical results suggested that increased TMB level in circulating tumor DNA (ctDNA) is associated with response to immunotherapy in NSCLC [13], suggesting that circulating TMB may provide useful and important information comparable to TMB in tissue or even better. The study showed that a TMB ctDNA $\geq 16$ is an independent predictor of response to therapy and the cut-off is technically robust enough to be used as a threshold in NSCLC. Interestingly, TMB ctDNA was not correlated with PD-L1 expression. The use of circulating biomarkers, instead of tissue, makes the TMB analysis an important alternative for patients with NSCLC who are not able to undergo a biopsy or whose tumor tissue is scant or unavailable.

The expression of PD-L1 and other chemokines involved in immune response have been widely investigated in liquid biopsy, confirming the advantages and the quality of an analysis based on circulating biomarkers, able to capture tumor dynamics [14-16]. Circulating tumor cells (CTCs) have been used to characterize tumor and PD-L1 dynamics during treatment, confirming that a considerable heterogeneity exists in PD-L1 expression in CTCs from NSCLC patients and that an increase of PD-L1-positive CTCs may predict resistance to checkpoints inhibitors [17]. Moreover, ctDNA dynamics has been successfully used to monitor immunotherapy response during treatment in different solid tumors, demonstrating that undetectable ctDNA at first clinical evaluation or a decrease of its concentration are associated with response and survival to immunotherapy. The analysis of ctDNA performed few weeks after treatment initiation may be a reliable biomarker for early assessment of treatment efficacy in patients treated with immunotherapy [18].

The application of circulating biomarkers to select patients for immunotherapy administration still needs to be further standardized, but the availability of robust and reproducible procedures for circulating biomarker analysis will provide a powerful tool in the era of personalized immunotherapy. Many different sources for these circulating biomarkers have been investigated: CTCs, ctDNA and exosomes. While CTCs may help in a better morphological characterization and enumeration of cell clones, ctDNA can be more reliable to reflect tumor burden and can be used to detect mutations, to better understand tumor heterogeneity and track tumor evolution [19]. Exosomes have an important role in cancer signaling, being involved in cell communication, and, more importantly, in immune escape, and can therefore act as valuable biomarkers [20].

In conclusion, it is likely that a reliable predictive biomarker for immunotherapy will be a multivariate model, composed of TMB, TILs, PD-L1 expression not only in tissue, but also as circulating biomarkers to select and monitor patients during treatment. The assessment of tumor genomic landscape during treatment has demonstrated that clonal evolution is driven by therapy-dependent immunoediting and that anti-PD-(L) 1 therapy is able to upregulate and modify the transcriptional activity of several genes in response to treatment. In this regard, the analysis of circulating biomarkers could offer a valuable, minimally invasive opportunity to monitor and characterize the genomic profile of tumors, with promising applications to predict response to immunotherapy.

Financial \& competing interests disclosure

M Del Re participated as speaker's bureau for Celgene, Janssen, Sanofi; participated in advisory board for Astellas, AstraZeneca; was a consultant for Ipsen, Sanofi; was a speaker for Astellas, AstraZeneca, Sanofi, Celgene, Novartis, Pfizer, Bio-Rad. A Addeo participated in advisory board for MSD Oncology, Roche, Takeda, Pfizer, Bristol-Myers Squibb, AstraZeneca, has a consulting role for MSD Oncology, Roche, received research grants for Boehringer Ingelheim. R Danesi participated in advisory board and had a consulting relationship with Ipsen, Novartis, Pfizer, Sanofi Genzyme, AstraZeneca, Janssen, Gilead; received travel, accommodation and expenses reimbursement from Ipsen, Sanofi Genzyme. The authors have no other relevant affiliations or financial involvement with any organization or entity with a financial interest in or financial conflict with the subject matter or materials discussed in the manuscript apart from those disclosed.

No writing assistance was utilized in the production of this manuscript. 


\section{References}

1. Fares CM, Van Allen EM, Drake CG, Allison JP, Hu-Lieskovan S. Mechanisms of resistance to immune checkpoint blockade: why does checkpoint inhibitor immunotherapy not work for all patients? Am. Soc. Clin. Oncol. Educ. Book 39, 147-164 (2019).

2. Reck M, Rodriguez-Abreu D, Robinson AG et al. Pembrolizumab versus chemotherapy for PD-L1-positive non-small-cell lung cancer. N. Engl. J. Med. 375(19), 1823-1833 (2016).

3. Ribas A, Hamid O, Daud A et al. Association of pembrolizumab with tumor response and survival among patients with advanced melanoma. JAMA 315(15), 1600-1609 (2016).

4. Dudley JC, Lin MT, Le DT, Eshleman JR. Microsatellite instability as a biomarker for PD-1 blockade. Clin. Cancer Res. 22(4), 813-820 (2016).

5. Jacquelot N, Roberti MP, Enot DP et al. Immunophenotyping of Stage III melanoma reveals parameters associated with patient prognosis. J. Invest. Dermatol. 136(5), 994-1001 (2016).

6. Yarchoan M, Hopkins A, Jaffee EM. Tumor mutational burden and response rate to PD-1 inhibition. N. Engl. J. Med. 377(25), 2500-2501 (2017).

7. Schumacher TN, Schreiber RD. Neoantigens in cancer immunotherapy. Science 348(6230), 69-74 (2015).

8. Le DT, Durham JN, Smith KN et al. Mismatch repair deficiency predicts response of solid tumors to PD-1 blockade. Science 357(6349), 409-413 (2017).

9. McGranahan N, Swanton C. Neoantigen quality, not quantity. Sci. Transl. Med. 11(506), pii:eaax7918 (2019).

10. Liu Y, Dong Y, Kong L, Shi F, Zhu H, Yu J. Abscopal effect of radiotherapy combined with immune checkpoint inhibitors. J. Hematol. Oncol. 11(1), 104 (2018).

11. Gainor JF, Shaw AT, Sequist LV et al. EGFR mutations and ALK rearrangements are associated with low response rates to PD-1 pathway blockade in non-small-cell lung cancer: a retrospective analysis. Clin. Cancer Res. 22(18), 4585-4593 (2016).

12. Riaz N, Havel JJ, Makarov V et al. Tumor and microenvironment evolution during immunotherapy with nivolumab. Cell 171(4), 934-949 (2017).

13. Gandara DR, Paul SM, Kowanetz M et al. Blood-based tumor mutational burden as a predictor of clinical benefit in non-small-cell lung cancer patients treated with atezolizumab. Nat. Med. 24(9), 1441-1448 (2018).

14. Chen G, Huang AC, Zhang W et al. Exosomal PD-L1 contributes to immunosuppression and is associated with anti-PD-1 response. Nature 560(7718), 382-386 (2018).

15. Del Re M, Marconcini R, Pasquini G et al. PD-L1 mRNA expression in plasma-derived exosomes is associated with response to anti-PD-1 antibodies in melanoma and NSCLC. Br. J. Cancer 118(6), 820-824 (2018).

16. Medrano RFV, Hunger A, Mendonca SA, Barbuto JaM, Strauss BE. Immunomodulatory and antitumor effects of type I interferons and their application in cancer therapy. Oncotarget 8(41), 71249-71284 (2017).

17. Janning M, Kobus F, Babayan A et al. Determination of PD-L1 expression in circulating tumor cells of NSCLC patients and correlation with response to PD-1/PD-L1 inhibitors. Cancers (Basel) 11(6), 835 (2019).

18. Forschner A, Battke F, Hadaschik D et al. Tumor mutation burden and circulating tumor DNA in combined CTLA-4 and PD-1 antibody therapy in metastatic melanoma - results of a prospective biomarker study. J. Immunother. Cancer 7(1), 180 (2019).

19. Snyder A, Morrissey MP, Hellmann MD. Use of circulating tumor DNA for cancer immunotherapy. Clin. Cancer Res. doi:10.1158/1078-0432.CCR-18-2688 (2019) (Epub ahead of print).

20. Liu Y, Gu Y, Cao X. The exosomes in tumor immunity. Oncoimmunology 4(9), e1027472 (2015). 
